\title{
Waveform simulation based on 3D dose distribution for acoustic wave generated by proton beam irradiation
}

\author{
Toshiyuki Terunuma ${ }^{\text {a) }}$ and Takeji Sakae \\ Proton Medical Research Center (PMRC), University of Tsukuba, 1-1-1 Ten-nohdai, \\ Tsukuba 305-8575, Japan \\ Yoshinori Hayakawa \\ Department of Biomedical Engineering, Toin University of Yokohama, 1614 Kurogane-cho, \\ Aoba-ku, Yokohama 225-8502, Japan \\ Akihiro Nohtomi \\ Atomic Energy Research Institute, Kinki University, 3-4-1, Kowakae, Higashi-Osaka-shi, \\ Osaka 577-8502, Japan \\ Yoshihisa Takada and Kiyoshi Yasuoka \\ Proton Medical Research Center (PMRC), University of Tsukuba, 1-1-1 Ten-nohdai, \\ Tsukuba 305-8575, Japan
}

\begin{abstract}
Akira Maruhashi
Department of Radiation Life Science and Radiation Medical Science, Research Reactor Institute, Kyoto University, Noda, Kumatori-cho, Sennan-gun, Osaka 590-0494, Japan
\end{abstract}

(Received 27 March 2006; revised 11 July 2007; accepted for publication 11 July 2007; published 24 August 2007)

A pulsed proton beam is capable of generating an acoustic wave when it is absorbed by a medium. This phenomenon suggests that the acoustic waveform produced may well include information on the three-dimensional (3D) dose distribution of the proton beam. We simulated acoustic waveforms by using a transmission model based on the Green function and the 3D dose distribution. There was reasonable agreement between the calculated and measured results. The results obtained confirm that the acoustic waveform includes information on the dose distribution. (C) 2007 American Association of Physicists in Medicine. [DOI: 10.1118/1.2767985]

Key words: acoustic waveform, 3D dose distribution, proton beam therapy, imaging plate

\section{INTRODUCTION}

Proton beam therapy is becoming an increasingly important tool because it is capable of producing a spatially localized maximum-dose region that can be utilized for the selective irradiation of a tumor. The depth of the peak dose depends on the beam energy and the tissue composition along the beam path. To ensure that the peak dose region is centered on the tumor in a patient, high accuracy is required in obtaining computed tomography images, in the treatment planning system, and irradiation equipment that are used and also in patient setup. Any errors might result in delivering an excessive dose to healthy tissue or an insufficient dose to the tumor. The most reliable verification for accurate irradiation is realtime in vivo imaging of the dose distribution in the patient during treatment. This is the goal of quality assurance for the proton beam therapy.

Some incident radiation beams, especially pulsed proton beams, generate an acoustic wave inside a medium. ${ }^{1-8}$ It has been reported that the amplitude of the acoustic signal is proportional to the beam current from a macroscopic viewpoint. Sulak et al. ${ }^{1}$ investigated the dependence of the signal amplitude on the beam diameter. However, from their results it is not possible to fully characterize the acoustic waveform, since the depth-dose distribution of protons (known as the Bragg peak) is too complex to be characterized only by a beam current or a beam diameter. We approached the problem from a microscopic rather than a macroscopic viewpoint and assumed that the acoustic pressure generated in a local region is proportional to the local energy deposited by the proton dose distribution. Based on this assumption, it is reasonable to consider that the acoustic waveform contains information on the three-dimensional (3D) dose distribution. The shape of the acoustic wave can be calculated by summing the pressures which propagated from each local point to the measurement point. To verify the validity of this assumption, we calculated the acoustic waveform by using a transmission model which uses the Green function and the 3D dose distribution. We then compared the results obtained using this model with actual experimental measurements.

\section{METHODS AND MATERIALS}

\section{II.A. Detection of the acoustic wave}

Detection of the acoustic waves was carried out in the vertical beam line at the branch facility of the Proton Medical Research Center, University of Tsukuba in the High Energy Accelerator Research Organization (KEK). The proton beam delivered from a $500 \mathrm{MeV}$ booster-synchrotron was degraded down to $230 \mathrm{MeV}$ by passing through a carbon energy degrader. Therefore, the energy distribution of protons and the width of the Bragg peak spread more than a 


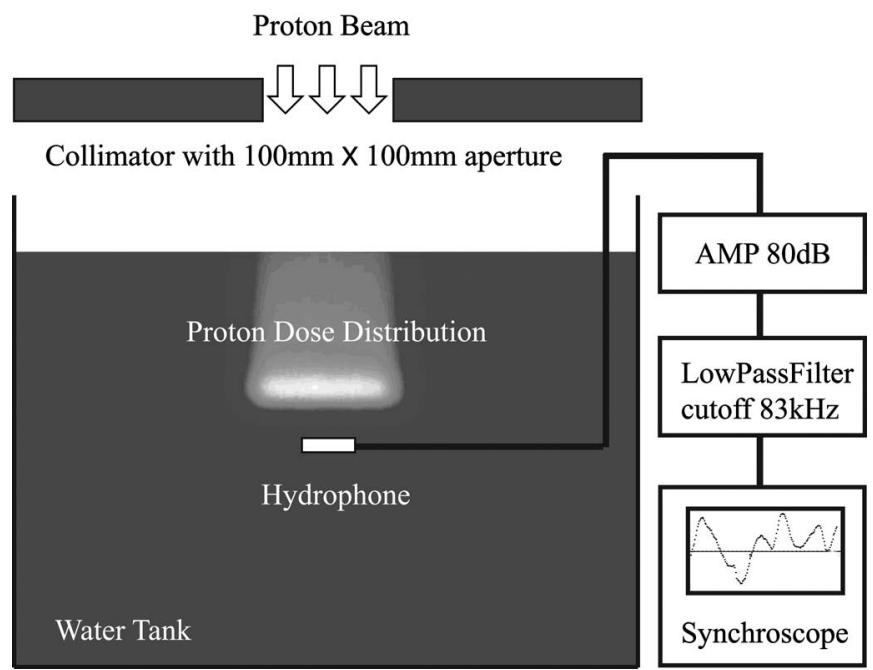

FIG. 1. Schematic diagram of the experimental setup.

monochromatic beam having the same range. The width and interval of the beam pulses were $50 \mathrm{~ns}$ and $50 \mathrm{~ms}$, respectively. To obtain up to $200 \mathrm{~mm} \times 200 \mathrm{~mm}$ irradiation field of uniform intensity at therapy table, proper combinations of the scatterer and the ring scatterer were selected. ${ }^{4,6,9}$ The field uniformity of the open beam was controlled within $\pm 2.5 \%$ error for treatment. In this experiment, the positions of the Bragg peak in water were variable over the range of 5-65 mm using a binary range shifter made of ABS resin. The absorbed dose rate in water at the Bragg peak was approximately $4 \mathrm{mGy} /$ pulse.

Figure 1 shows a schematic diagram of experimental setup. A Langevin type hydrophone (model ST-8005, Oki Electric industry Co., Ltd.) was used to detect a train of acoustic pulses generated by the pulsed proton beams. The shape of the hydrophone is a cylinder of $15 \mathrm{~mm}$ in length and $6 \mathrm{~mm}$ in diameter (embedded in a rubber sheath of 10 $\mathrm{mm}$ in diameter). The sensitivity of the hydrophone is axially homogeneous, and is $-200 \mathrm{~dB}$ in the frequency range between $100 \mathrm{~Hz}$ and $70 \mathrm{kHz}$, where the sensitivity $0 \mathrm{~dB}$ corresponds to $1 \mathrm{~V} / \mathrm{mPa}$. The hydrophone was placed at depths of 90 or $140 \mathrm{~mm}$ in water. Detected signals were amplified 10000 times and filtered by a low-pass filter (LPF) for noise cut, with a cutoff frequency of $83 \mathrm{kHz}$, before being accumulated 1000 times and averaged in a digital synchroscope (type 9400, LeCroy Co., Ltd.). The synchroscope was triggered by the "kicker pulses" of the proton synchrotron which were synchronized with the time of beam extraction.

\section{II.B. Calculation of 3D dose distribution from measured 2D dose distribution}

Since the time resolution of acoustic detection $(1 \mu \mathrm{s})$ results in a limited spatial resolution (estimated to be $1.5 \mathrm{~mm}$ by taking the speed of sound in water to be $1500 \mathrm{~m} / \mathrm{s}$ ), we need to achieve 3D dose measurements having at least the same resolution. Scanning a pin-point detector was judged to be a very time-consuming method for measuring the
Proton Beam
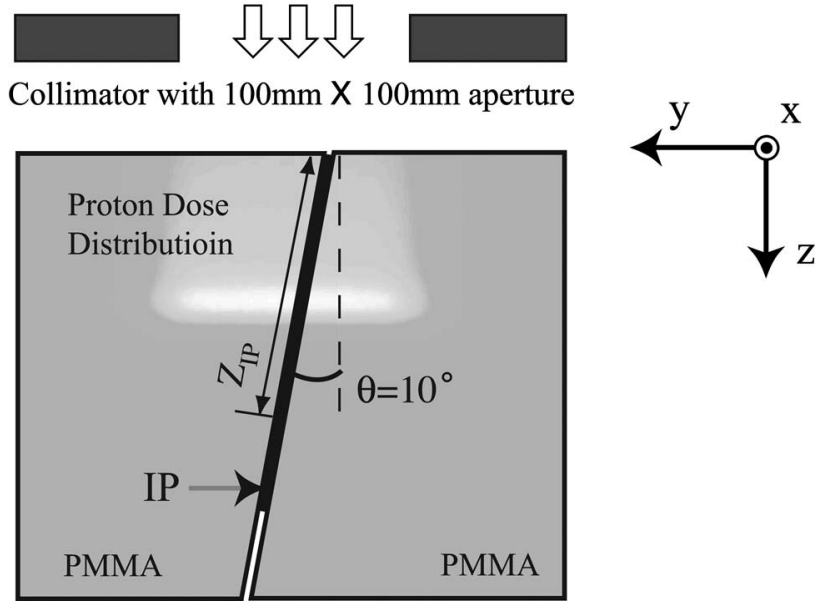

FIG. 2. 2D measurement method of symmetric proton beam irradiation. The IP was inserted into the gap between the two oblique edges formed by PMMA holders.

approximately 135 million points in a $200 \mathrm{~mm} \times 200 \mathrm{~mm}$ $\times 100 \mathrm{~mm}$ field. Instead, we chose to use an imaging plate (IP) which is widely used to detect two-dimensional (2D) images formed by ionizing radiation. ${ }^{10-13}$ By creating an axially symmetric dose distribution about the beam axis using a collimator having a $100 \mathrm{~mm} \times 100 \mathrm{~mm}$ aperture, it was possible to detect a 2D dose distribution using the IP and then calculate the 3D dose distribution from it.

The setup for the IP measurement is shown in Fig. 2. An IP (BAS-III 2025, Fuji Film Co., Ltd.) having dimensions $200 \mathrm{~mm} \times 250 \mathrm{~mm}$ was sandwiched between two slanted $\left(\theta=10^{\circ}\right)$ polymethylmethacrylate (PMMA) holders to prevent the IP from affecting the proton range. The stopping power of PMMA relative to water is $1.16 .^{12}$ The equivalent depth in water $Z_{\text {water }}$ can be calculated using the following equation: $^{12}$

$$
Z_{\text {water }}=1.16 Z_{\mathrm{IP}} \cos \theta
$$

where $Z_{\mathrm{IP}}$ is the depth in the slanted plane of the PMMA obtained by measuring the profile on the IP.

A Cartesian coordinate system $(x, y, z)$ was used in this study. The $x$ and $y$ directions are perpendicular to the beam axis while the $z$ coordinate is the depth in water in the direction of beam. Although the measured dose image of the slanted IP $D_{2 \mathrm{D}}\left(x, z_{\mathrm{IP}}\right)$ did not lie in the $x-z$ plane, the image $D_{2 \mathrm{D}}\left(x, z_{\text {water }}\right)$ could be regarded as lying in the $x-z$ plane since the distribution was laterally almost uniform over the area of the IP. The uniformity of the collimated $100 \mathrm{~mm}$ $\times 100 \mathrm{~mm}$ field was estimated within about $\pm 1.25 \%$ error by the uniformity of the open beam. Therefore, in this axially symmetric distribution case, the $3 \mathrm{D}$ dose matrix $D_{3 \mathrm{D}}(x, y, z)$ was calculated from the following equation:

$$
D_{3 \mathrm{D}}(x, y, z)=\frac{D_{2 \mathrm{D}}(x, z) D_{2 \mathrm{D}}(y, z)}{D_{2 \mathrm{D}}(b, z)},
$$

where $b$ is the point corresponding to the position of the beam axis and is equal to $x / 2$. 


\section{II.C. Acoustic wave transmission model}

The acoustic pressure $p\left(\boldsymbol{r}^{\prime}, t\right)$ propagated from an inhomogeneous source is given by the following equation which means summation of the Green function: ${ }^{1,3,7}$

$$
p\left(\boldsymbol{r}^{\prime}, t\right)=\frac{\alpha}{4 \pi C_{p}} \int \frac{d \boldsymbol{V}}{R} \frac{\partial^{2}}{\partial t^{2}} q\left(\boldsymbol{r}, t-\frac{R}{c}\right),
$$

where $q(\boldsymbol{r}, t)$ is the excess heat per unit volume at the source point $\boldsymbol{r}(x, y, z), c$ is the speed of sound, $\alpha$ is the thermal expansion coefficient, $C_{p}$ is the specific heat capacity of the medium, and

$$
R=\left|\boldsymbol{r}-\boldsymbol{r}^{\prime}\right|=\sqrt{\left(x-x^{\prime}\right)^{2}+\left(y-y^{\prime}\right)^{2}+\left(z-z^{\prime}\right)^{2}}
$$

is the distance from the source to an observation point $\boldsymbol{r}^{\prime}\left(x^{\prime}, y^{\prime}, z^{\prime}\right)$.

Since the beam pulse width $\left(50 \times 10^{-9} \mathrm{~s}\right)$ and the stopping time for $\mathrm{MeV}$ protons in a medium $\left(\sim 10^{-10} \mathrm{~s}\right)$ are much shorter than the acquisition time for an acoustic wave $\left(\sim 10^{-5} \mathrm{~s}\right)$, the energy deposition can be considered to be instantaneous. Thus, the heating rate can be regarded as being proportional to the dose deposition ${ }^{7}$

$$
\dot{q}(\boldsymbol{r}, t) \propto D(\boldsymbol{r}) \delta(t)
$$

where $D(\boldsymbol{r})$ is the dose deposition and $\delta$ is the Dirac delta function. Therefore, Eq. (3) is proportional to the expression

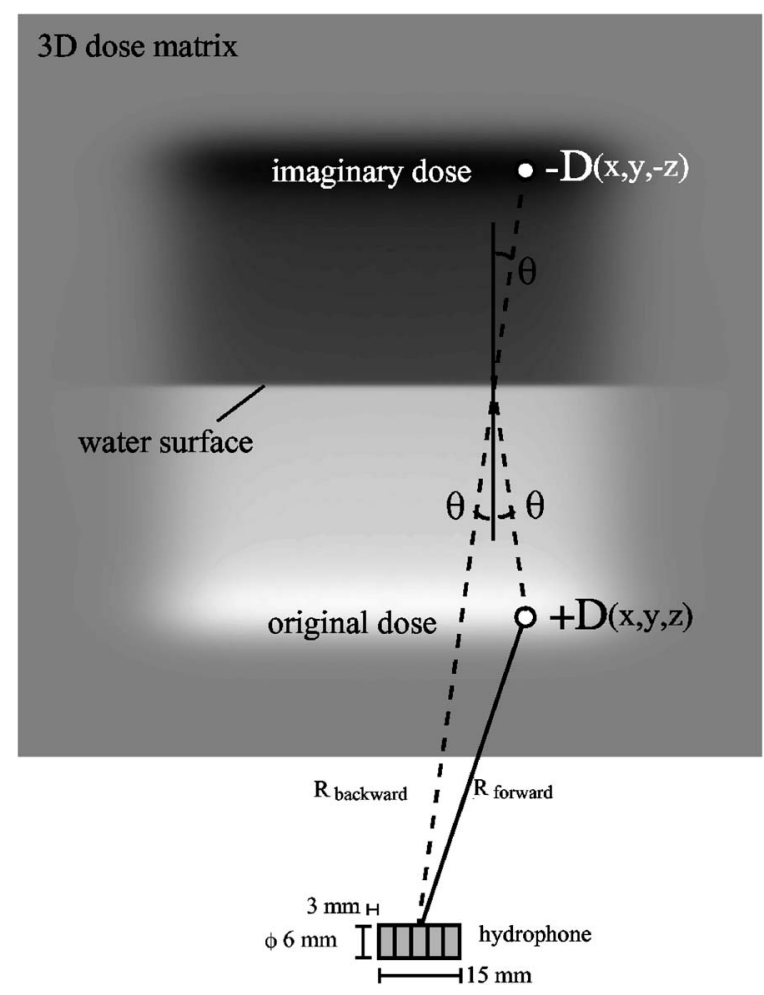

FIG. 3. Schematic image of the pressure calculation. An imaginary component of 3D dose distribution is added above the water surface of the original 3D dose distribution. given below and we can calculate the waveform using the $3 \mathrm{D}$ dose data

$$
p\left(\boldsymbol{r}^{\prime}, t\right) \propto \frac{\alpha}{4 \pi C_{p}} \frac{\partial}{\partial t} \int \frac{d \boldsymbol{V}}{R} D(\boldsymbol{r}) \delta\left(t-\frac{R}{c}\right) .
$$

The attenuation effect of sound in water is neglected in Eq. (6) since the $1 / e$ attenuation length is estimated to be over $400 \mathrm{~km}$ for the frequency range of acoustic waves.

The wave that was reflected at the water surface was observed in the experiment. To reproduce the acoustic waveform, a hypothetical negative 3D dose distribution was introduced above the surface (see Fig. 3), since the sign of the pressure is reversed on reflection at the free surface. ${ }^{14}$ The reflected wave at the wall of tank was ignored because the distance from hydrophone to the wall $(300 \mathrm{~mm})$ was over two times longer than that to the water surface (90 or 140 $\mathrm{mm})$. Therefore, two waves from both boundaries were separated perfectly. We were not concerned with observing the waves reflected from the wall in this study.

\section{RESULTS AND DISCUSSIONS}

\section{III.A. Calculation of 3D dose distribution from measured 2D dose distribution}

Two-dimensional dose distributions for several proton energies were measured to a spatial resolution of $0.80 \mathrm{~mm}$ spatial resolution using an IP. The resolution in the $z$ direction was $0.92 \mathrm{~mm}$ when the measurement depth in PMMA was converted into a water-equivalent depth using Eq. (1).

Photostimulated luminescence (PSL) signal detected by an IP is not proportional to a depth-dose profile of a proton beam detected by an ionization chamber in the high linearenergy-transfer region due to quenching effects. ${ }^{11,12}$ The quenching factor which is defined as a decrease ratio of PSL signal to the dose is depth-dependent obviously. ${ }^{11}$ The quenching factor depends on the depth-dependent energy distribution of protons. Thus modulating a quenching factor measured for a monochromatic proton beam makes it possible to calculate briefly the depth-dose curve for the energyspread proton beam at KEK. A $125 \mathrm{MeV}$ proton beam, which range is $87 \mathrm{~mm}$ at the proton synchrotron in the hospital of university of Tsukuba, was selected as a monochromatic proton beam. The depth-dependent quenching factor was measured by the IPs and a parallel-plate ionization chamber (PPIC). The quenching factor at the Bragg peak is $11.9 \%$. As shown in Fig. 4, several PSL curves, which have different ranges, were calculated by shifting and modulating the PSL curve for $125 \mathrm{MeV}$ protons. The modulation weights of PSL curves were optimized by the least square method to fit the stacked PSL curve to the PSL curve measured at KEK. These modulation weights and several range-shifted depth-dose curves for the $125 \mathrm{MeV}$ proton beam provided the virtual depth-dose curve, corresponding to the depth-dose curve 


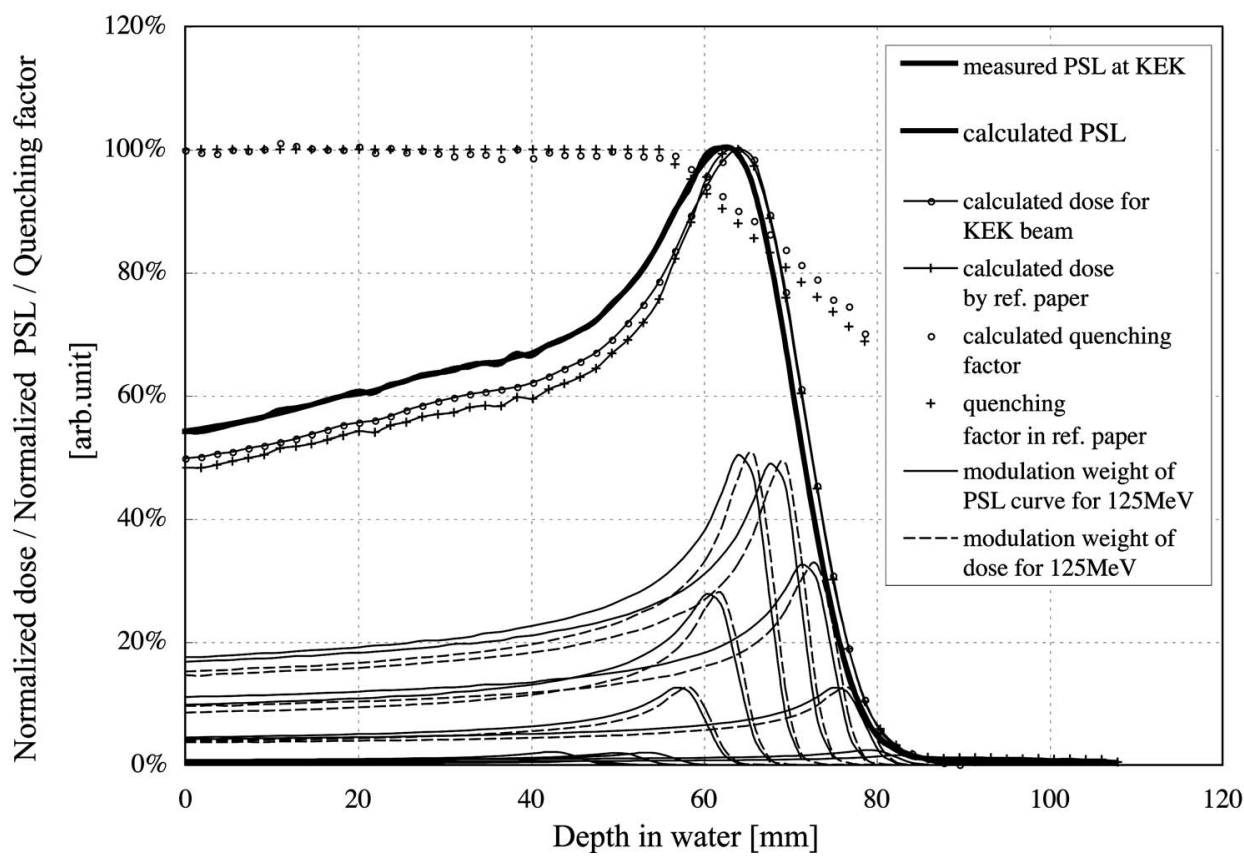

FIG. 4. Depth dose and quenching factor. The amount of the modulation weights of range-shifted PSL curves and depth doses from $125 \mathrm{MeV}$ proton beam are relative. As a reference, the quenching factor, which was simply scaled down from Ref. 11, and the calculated dose by $10 \%$ quenching factor at the Bragg peak were shown.

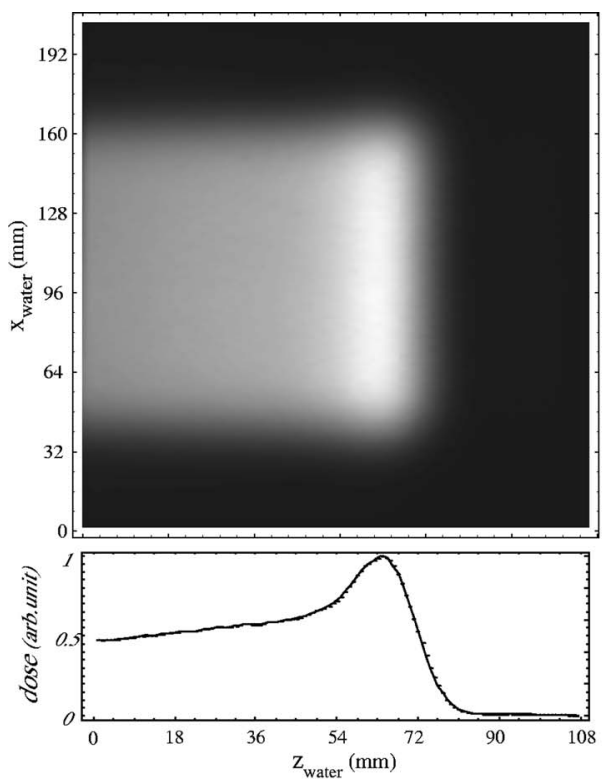

(a)
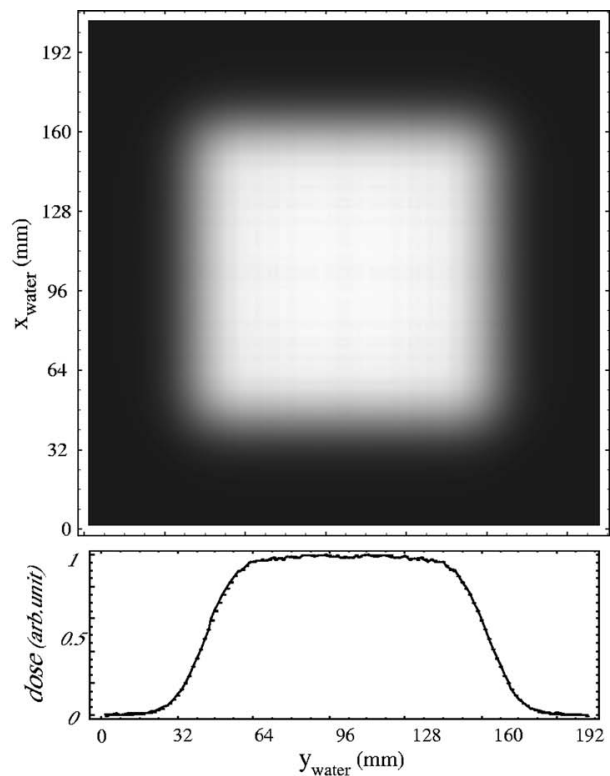

(b)

FIG. 5. Calculated 3D dose distribution for the Bragg curve: (a) Image in the $x-z$ plane and depth dose profile on beam axis and (b) image in the $x-y$ plane and lateral dose profile at the Bragg peak. Profiles before and after 3D calculation are represented by solid lines and dotted lines, respectively.

measured by PPIC at KEK, and the quenching factor for energy-spread proton beam. Figure 4 shows the result. The quenching factor calculated at the Bragg peak is $8.0 \%$ in the error of $1 \%$. However, it must be reminded that this correction is an approximation.

The 3D dose distributions were calculated by the 2D dose distributions. Figures 5(a) and 5(b) show the results obtained.

\section{III.B. Calculation of the acoustic waveforms}

As shown in Fig. 3, the imaginary components of the $3 \mathrm{D}$ dose distributions were added above the water surface of the original 3D dose distributions in order to calculate the effect of pressure reflection at the water surface. The dimensions of the 3D matrices for $D_{3 \mathrm{D}}(x, y, z)$ are 256 $\times 256 \times 240$. 


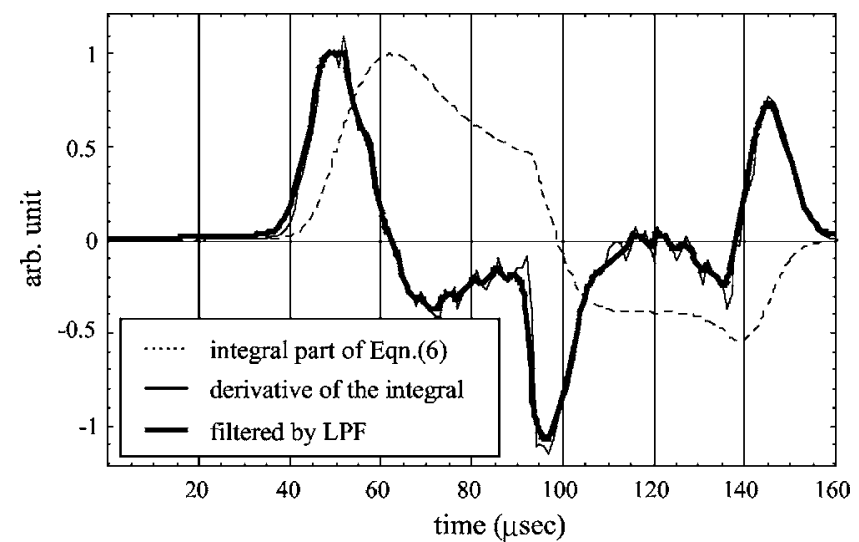

FIG. 6. Steps of waveform calculation and results.

At first, using these 3D dose data, the integral part of Eq. (6) was calculated by summing it by the Green function proportional to the local dose as a function of the traveling time to the hydrophone position (see Fig. 6). Secondary, the waveform was obtained by taking the derivative of the integral. The effect of the LPF for noise cut was simulated by multiplying the Fourier-transformed waveform by the frequency function of the LPF and carrying out the inverse Fourier transform.

To calculate the acoustic waveform strictly in the processes mentioned above, the influence of the point-detector assumption on the calculation should be considered. Three waveforms are shown in Fig. 7 for (a) one point at the center of the detector, (b) five distributed points in axis of the detector and averaged them, and (c) 300 distributed points and averaged them, in which the size of each element is equal to the voxel size of 3D dose matrix. Since there are no significant differences, we adopted method (b).

Figure 8 shows influence of the correction for the PSL quenching on the waveform. The influence of the estimation error for the correction is as small as $1 \%$.

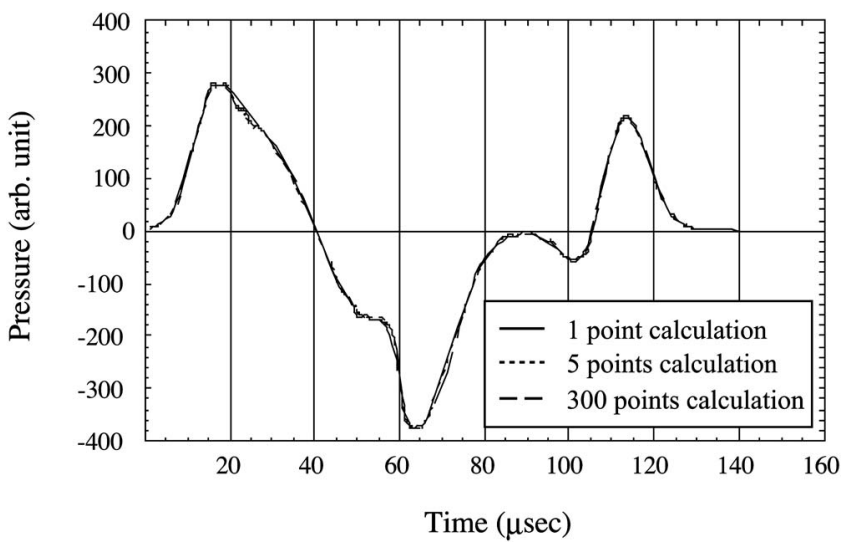

FIG. 7. Acoustic waveforms calculated for one point at the center of the detector, for distributed five points in axis of the detector and for distributed 300 points in which the size of each element is equal to the voxel size of 3D dose matrix. This calculation was done for the beam at the Bragg peak of 65 $\mathrm{mm}$ depth and for the hydrophone at $90 \mathrm{~mm}$ depth.

Calculation results with measured waveforms are shown in Fig. 9. Figures 9(a)-9(c) show the influence of the proton range. Figures 9(d)-9(f) show the influence of off-axis measurement on the waveform. Figures 9(a) and 9(d) show the influence of detector position also. The calculated results are in good agreement to the experimental results in all cases. It is proven that the timing structure of acoustic wave can be calculated very well from the $3 \mathrm{D}$ dose distributions. There is validity in our assumption that the acoustic pressure generated in a local region is proportional to the local energy deposited. Thus the acoustic waveform contains information on the $3 \mathrm{D}$ dose distribution.

However, a quantitative discussion is difficult from these results. If a perfect $3 \mathrm{D}$ reconstruction method was applied to this inverse problem, the quantitative comparison would be performed. However, in general, it is necessary to position a very large number of detectors in an enclosed 3D space since the acoustic signal is emitted in all directions and this is far from straightforward to do.
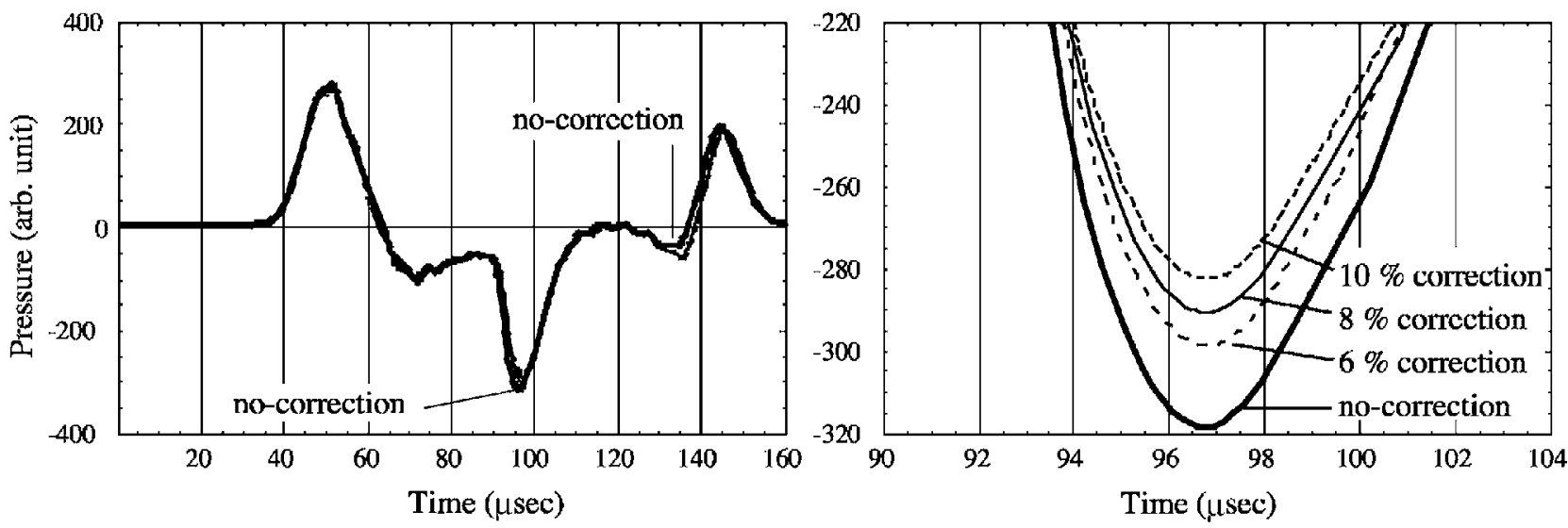

FIG. 8. Influence of the correction factor of the PSL quenching on the calculated acoustic waveform. waveforms were calculated for $6 \%$, $10 \%$, the adequate $8 \%$ correction, and no-correction at the Bragg peak. These calculations were done for the beam with the Bragg peak of 65 mm depth and for the hydrophone at $140 \mathrm{~mm}$ depth. 

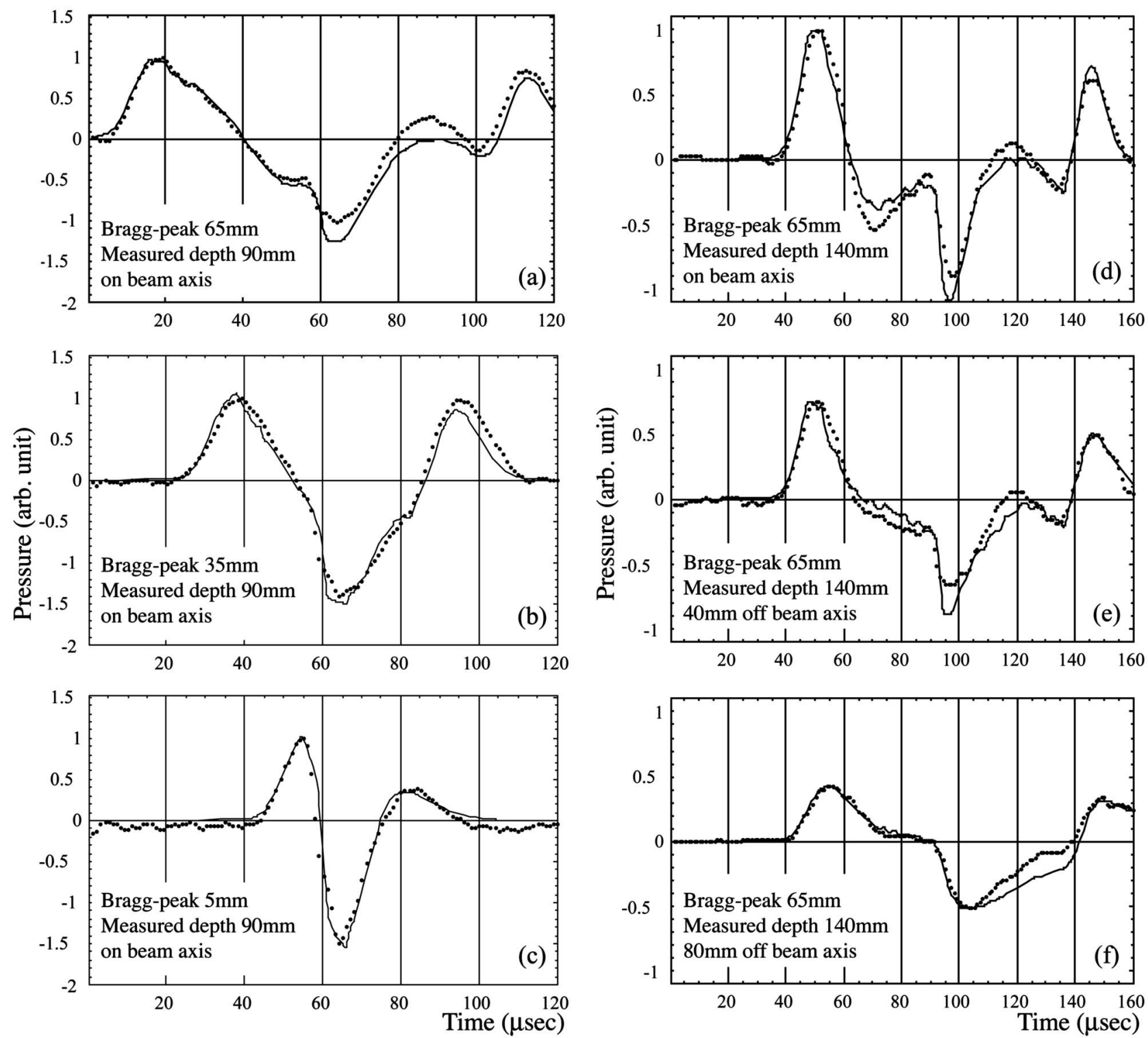

FIG. 9. Influence of the proton range on the waveform [(a), (b), (c)] and influence of off-axis measurement on the waveform [(d), (e), (f)]. Solid lines indicate the calculated pressure waveforms, while the dotted lines indicate the experimental waveforms detected by the hydrophone in water. waveforms (a), (b), and (c) were normalized using the first maximum. waveforms (d), (e), and (f) were normalized using the first maximum of the on-axis measurement.

Hayakawa et al. ${ }^{8}$ have reported that the acoustic signal could also be measured at the surface of the patient's body when it is irradiated by the pulsed proton beam during treatment. This holds out the possibility of verifying the dose distribution during treatment, although several difficulties (such as the scattering and the absorption of the acoustic wave in vivo) would need to be overcome before this could become a reality.

\section{CONCLUSION}

Acoustic waveforms have been calculated from 3D dose distributions using a transmission model based on the Green function. In all cases, the calculated results agreed well with the experimental results. These results confirm that the acoustic waveform includes information on 3D dose distribution.
${ }^{a)}$ Electronic mail: terunuma @ pmrc.tsukuba.ac.jp

${ }^{1}$ L. Sulak et al., "Experimental studies of the acoustic signature of proton beams traversing fluid media," Nucl. Instrum. Methods 161, 203-217 (1979).

${ }^{2}$ G. A. Askariyan, B. A. Dolgoshien, A. N. Kalinovsky, and N. V. Mokhov, "Acoustic detection of high energy particle showers in water," Nucl. Instrum. Methods 164, 267-278 (1979).

${ }^{3}$ G. E. Sieger and H. W. Lefevre, "Time-resolved measurement of acoustic pulses generated by $\mathrm{MeV}$ protons stopping in aluminum," Phys. Rev. A 31, 3929-3936 (1985).

${ }^{4}$ Y. Hayakawa, J. Tada, T. Inada, T. Wagai, and K. Yoshioka, "Acoustic pulse generation in water by pulsed proton beam irradiation and its possible application to radiation therapy," Jpn. J. Appl. Phys. 28, 217-219 (1989).

${ }^{5}$ T. Bowen, C. X. Chen, S. C. Liew, W. R. Lutz, and R. L. Nasoni, "Observation of ultrasonic emission from edges of therapeutic X-ray beams," Phys. Med. Biol. 36, 537-539 (1991).

${ }^{6}$ J. Tada, Y. Hayakawa, K. Hosono, and T. Inada, "Time resolved properties of acoustic pulses generated in water and in soft tissue by pulsed 
proton beam irradiation-A possibility of doses distribution monitoring in proton radiation therapy," Med. Phys. 18, 1100-1104 (1991).

${ }^{7}$ N. A. Baily, "A review of the processes by which ultrasound is generated through the interaction of ionizing radiation and irradiated materials: Some possible applications," Med. Phys. 19, 525-532 (1992).

${ }^{8}$ Y. Hayakawa, J. Tada, N. Arai, K. Hosono, M. Sato, T. Wagai, H. Tsuji, and $\mathrm{H}$. Tsujii, "Acoustic pulse generated in a patient during treatment by pulsed proton radiation beam," Radiat. Oncol. Investig. 3, 42-45 (1995). ${ }^{9}$ PARMS, Biomedical Research Facility, KEK Annual Report, 1982, pp. 139-143.

${ }^{10}$ Y. Iwabuchi, N. Mori, K. Takahashi, T. Matsuda, and S. Shioyama, "Mechanism of photostimulated luminescence process in $\mathrm{BaFBr}: \mathrm{Eu}^{2+}$ phosphors," Jpn. J. Appl. Phys., Part 1 33, 178-185 (1994).

${ }^{11}$ A. Nohtomi, T. Terunuma, R. Kohno, Y. Takada, Y. Hayakawa, A. Maru- hashi, T. Sakae, and K. Yasuoka, "Response characteristics of an imaging plate to clinical proton beams," Nucl. Instrum. Methods Phys. Res. A 424, 569-574 (1999).

${ }^{12}$ A. Nohtomi, T. Sakae, T. Terunuma, Y. Tsunashima, K. Hosono, and Y. Hayakawa, "Measurement of depth-dose distribution of protons by an imaging plate," Nucl. Instrum. Methods Phys. Res. A 511, 382-387 (2003).

${ }^{13}$ T. Terunuma, T. Sakae, A. Nohtomi and Y. Tsunashima, "The direct measurement using imaging plate for coincidence of radiation centre and laser position in external radiation therapy," Phys. Med. Biol. 48, N59-N63 (2003).

${ }^{14}$ R. P. Feynman, The Feynman Lecture on Physics (Addison-Wesley, New York, 1963), Vol. 1, Chap. 49-1. 